\title{
ACTA APPLICANDAE MATHEMATICAE
}

\section{An International Journal on Applying Mathematics \\ and Mathematical Applications}

Managing Editor:

MICHIEI HAZEWINKEL

Centre for Mathematics and Computer Science, Amsterdam

This journal is devoted to the art and techniques of applying mathematics and the development of new applicable mathematical theories. It contains papers on different aspects of the relation between theory and application: descriptive papers on actual applications, papers on technique and method when applying existing mathematical tools (e.g. in working examples), and of special importance, papers on mathematics motivated by the prospect of potential application, and on those established parts of mathematics which seem to be on the threshold of application. In particular, the editors hope to create a forum through which mathematicians and other scientists can be introduced to new techniques of applying mathematics and/or to new fields of application. This is achieved via the medium of longer survey, review and state-ofthe art papers which are written with a large and varied academic audience in mind.

Some Recent Articles

Charles J.K. Batty and Derek W. Robinson: Positive One-Parameter Semigroups on Ordered Banach Spaces. David E. Evans: Quantum Dynamical Semigroups, Symmetry Groups, and Locality. L. L. Helms: Order Properties of Attractive Spin Systems. A.M. Vinogradoy: Local Symmetries and Conservation Laws. A. Bensoussan: On the Theory of Option Pricing. Edward W. Kamen et aZ.: Pointwise Stability and Feedback Control of Linear systems with Noncommensurate Time Delays.

Subscription Information

ISSN $0167-8019$

1985, Volumes 3 and 4 (6 issues)

Institutional rate:

Dfl. $165,-/$ Us\$ 63.00 per volume incl.postage/handling Private rate: Dfl. $78,-/$ US\$ 29.50 per volume incl.postage/handling

Private subscriptions should be sent direct to the publishers.

\section{Reidel Publishing Company}

A Member of the Kluwer Academic Publishers Group

P.O. Box 17, 3300 AA Dordrecht, The Netherlands. 190 old Derby Street, Hingham MA 02043, U.S.A.

Falcon House, queen Square, Lancaster LAl IRN, U.K. 


\section{EDITORIAL POLICY}

The BULLETIN of the Australian Mathematical Society aims at quick publication of original research in all branches of mathematics. The Editors receive about twice as much material as can be published in the BULLETIN; many meritorious papers can, therefore, not be accepted. Manuscripts must be in their final, publishable form when submitted and papers which appear to need revision are unacceptable.

The BULLETIN is printed by a photo-offset process from a carefully prepared typescript. The type founts and special symbols available are accordingly restricted.

Authors can assist the Editors and improve their papers' chances of acceptance by observing some simple rules:

Submit 3 clean, high quality copies of the typescript with generous spacing and margins. We prefer clear and full arguments, and especially clear and full motivation, to mathematical telegrams. Make the style attractive and avoid ugly abbreviations.

Avoid all mathematical symbols in the title (even harmless looking ones like $R^{n}, L_{p} A_{n}$-algebra): they lead to unnecessary difficulties in the production processes, and are a great nuisance also after publication, in reviews, bibliographies, and so on. Arrange references alphabetically (by surname of first author), and ensure their accuracy: authors' names should appear as in the work quoted; and if this has not appeared yet or is not readily accessible to everybody (for example, a preprint), a photocopy of the title page of the copy you have used will be of great help to us.

Do not distribute your paper widely in preprint form as this damages its case. for fast publication.

\section{AUTHORS' ABSTRACTS}

1. Each paper must include an abstract of not more than 200 words.

2. In writing abstracts, authors should bear in mind that these may be the only parts of the papers that are read.

3. The abstract should contain a brief but informative summary of the contents of the paper, but no inessential details.

4. The abstract should be self-contained, but may refer to the title.

5. The abstract should be written in completely connected sentences, not as a list of headings. Abbreviations should be avoided.

6. The abstract should be non-technical, and intelligible without reference to the full paper. Specific references (by number) to a section, proposition, equation. bibliographical item should be avoided.

\section{SUBJECT CLASSIFICATION}

Authors should include in their papers one or more classification numbers. following the 1980 Mathematics Subject Classification. Details of this scheme can be found in each Annual Index of Mathematical Reviews.

\section{THE BULLETIN}

The BULLETIN of the Australian Mathematical Society began publication in 1969. Normally two volumes of three numbers are published annually. The BULLETIN is published for the Australian Mathematical Society by the Australian Mathematical Publishing Association Inc. through the University of Queensland 


\section{THE AUSTRALIAN MATHEMATICAL SOCIETY}

President:

R. S. Anderssen

Secretary: W. R. Bloom

Treasurer: B. D. Jones
Division of Mathematics and Statistics.

C.S.I.R.O., P.O. Box 1965 ,

Canberra City, A.C.T., 2601, Australia.

School of Mathematical and Physical Sciences,

Murdoch University,

P.O. Box 14, Willetton, W.A., 6155, Australia.

Department of Mathematics,

University of Queensland,

St. Lucia, Queensland, 4067, Australia.

(These officers serve until May 1986)

\section{Membership and correspondence}

Applications for membership, notices of change of address or title or position, and other correspondence, except as noted below, should be sent to the Secretary. Members' subscriptions and correspondence related to accounts should be sent to the Associate Treasurer. Correspondence about the distribution of the Society's BULLETIN, GAZETTE and JOURNAL, and orders for back numbers should be sent to the Treasurer.

\section{Other publications of the Society}

The JOURNAL of the Australian Mathematical Society began publication in 1959 and since 1975 has appeared in two series, Series A (Pure Mathematics and Statistics) and Series B (Applied Mathematics). The Editors are:

(A) Dr. T. E. Hall,

Department of Mathematics.

Monash University,

Clayton, Victoria 3168.

Australia.
(B) Professor E. O. Tuck,

Department of Applied Mathematics.

University of Adelaide,

G.P.O. Box 498, S.A. 5001.

Australia.

The Australian Mathematical Society GAZETTE started publication in 1974, and carries news items, mathematical articles of general interest, and articles on tertiary mathematics teaching. Manuscripts for publication should be sent to the Editor:

Dr. G. L. Cohen

School of Mathematical Sciences.

The New South Wales Institute of Technology.

Broadway, N.S.W. 2007.

Australia. 